\title{
The Rings of Jupiter
}

\author{
I. DE PATER, D. P. HAMilton, M. R. SHOWAlter, H. B. Throop, \\ AND J. A. BURNS
}

\section{Book Chapter}

This is Chapter 6 in: Planetary Ring Systems, Eds. M. S. Tiscareno and C. D. Murray. Cambridge University Press, Cambridge, UK. In Press (2017)

This version is free to view and download for personal use only. Not for re-distribution, re-sale or use in derivative works. Copyright: Cambridge University Press. For more information on the book, see: www.cambridge.org/9781107113824.

\subsection{Introduction}

The jovian ring was discovered as the result of a concerted search by the Voyager 1 cameras as the spacecraft passed Jupiter on March 4, 1979 (Smith et al. 1979b). Voyager's wide- and narrow-angle cameras were targeted at the ring plane and exposed for 11 minutes. The wide-angle field of view had complete coverage of the region, but was badly overexposed by glare from Jupiter. However, by good fortune, the co-aligned narrow-angle camera captured the tip of the ring. Subsequently, the Voyager 2 spacecraft performed a more detailed imaging sequence (Smith et al. 1979a). That sequence included views looking back toward the Sun while passing through Jupiter's shadow. In these views, what was hitherto a faint ring glowed brightly, indicating that the ring is dominated by micron-sized dust grains, which forwardscatter into a diffraction lobe just a few degrees wide. While today we take it for granted that the majority of planetary rings are faint and dusty, the jovian ring was the first ring found to be dominated by fine dust, and it remains an archetype for similar dynamical systems.

Hints of the ring's existence had previously been obtained in 1974, when Pioneer 11 detected "drop-outs" in the measurements of charged particles, attributed at the time to absorption by a potential ring around Jupiter (Fillius et al. 1975 van Allen et al. 1975). The rings have been subsequently imaged by three more spacecraft-Galileo, Cassini, and New Horizons - as well as by the Hubble Space Telescope (HST) and by several large, ground-based telescopes.

Jupiter's ring system consists of four principal components (Burns et al., 1984, Showalter et al., 1985, 1987 Ockert-Bell et al., 1999): a) the 'main ring' between $\sim 1.75$ $R_{\mathrm{J}}$ and $1.81 R_{\mathrm{J}}$ (where the Jovian radius $R_{\mathrm{J}}=71,492 \mathrm{~km}$ )

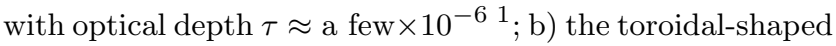
'halo ring' interior to the main ring, which is a radially confined torus of faint material with $\tau \approx 10^{-6}$; and c) the two faint 'gossamer rings' coinciding with the orbits of Amalthea and Thebe (both $\tau \sim 10^{-7}$; Figure 6.1 Table 6.1.

It is believed that the rings' optical depth is dominated by small grains (radius $r<\lambda$, with $\lambda$ the observing wavelength), which are produced by collisions or interactions with larger parent bodies ( $r \geq \lambda$, and perhaps up to hundreds of meters in size). These parent bodies have not been individually seen, but their existence is indirectly inferred by the rings' existence, and directly by measurements of the rings' phase curve and spectrum.

Since the jovian ring system is extremely faint, it is challenging to determine its precise structure and the nature of the ring particles. Information on the rings' physical properties can be derived by obtaining data over a range of phase angles $\alpha$ and wavelength $\lambda$; inversion of these spectra and phase curves constrain the size and composition of both dust and parent bodies.

Since a major component of the rings is micron-sized dust, and the lifetimes of such grains are brief due to the effects of solar radiation pressure, plasma and electromagnetic perturbations $\left(\sim 10^{3}-10^{5}\right.$ years for a $1 \mu \mathrm{m}$ grain, Burns et al. (2004)), the rings must be young and continually replenished with new material. Burns et al. (1999) suggested that impact ejecta from the inner (ring-moon) satellites provide the sources for this ring material. They showed that in particular the gossamer rings' morphology can be explained by ejecta lost from Amalthea and Thebe on their inclined orbits, spiraling inwards due to Poytning Robertson drag.

When rings are viewed edge-on (i.e., when the observer's elevation angle $B$ decreases to $\sim 0^{\circ}$ ), relatively flat, optically thick rings fade as a result of mutual shadowing and obscuration of ring particles. In contrast, the surface brightness of optically thin rings brightens as $1 / \sin |B|$, since the particles appear to be packed more closely as seen from the observer's perspective. Hence, images both of the unlit and (nearly) edge-on rings provide essential and complementary information to images taken under "normal" (back-scattered light) viewing conditions. For Jupiter, Earth-based telescopes, including HST, have imaged the rings in backscattered light at phase angles $0^{\circ}-\sim 11^{\circ}$ and wavelengths $0.5 \mu \mathrm{m}-2.2 \mu \mathrm{m}$, while spacecraft have provided data over the range $0^{\circ}$ -

1 Unless otherwise noted, $\tau$ always refers to the normal optical thickness. 
Table 6.1. Properties of Jupiter's ring system. ${ }^{a}$

\begin{tabular}{llllll}
\hline \hline & Halo $^{b}$ & Main ring & Amalthea ring & Thebe ring & Thebe extension \\
\hline Radial location $\left(\mathrm{R}_{J}\right)$ & $1.4-1.71$ & $1.72-1.806$ & $1.8-2.55$ & $1.8-3.10$ & $3.1-3.8$ \\
Radial location $(\mathrm{km})$ & $100000-122400$ & $122400-129100$ & $122400-181350$ & $122400-221900$ & $221900-270000$ \\
Vertical thickness & $\sim 5 \times 10^{4} \mathrm{~km}$ & $30-100 \mathrm{~km}$ & $\sim 2300 \mathrm{~km}$ & $\sim 8500 \mathrm{~km}$ & $\sim 9000 \mathrm{~km}$ \\
Normal optical depth & few $\times 10^{-6}$ & few $\times 10^{-6}$ & $\sim 10^{-7}$ & $\sim 10^{-8}$ & $\sim 10^{-9}$ \\
Particle size & $(\mathrm{sub}) \mu \mathrm{m}$ & broad distribution & broad distribution & broad distribution & \\
\hline \hline
\end{tabular}

${ }^{a}$ Table from de Pater and Lissauer (2015), based on data from Ockert-Bell et al. (1999), andde Pater et al. (1999, 2008).

${ }^{b}$ Numbers quoted are based upon the Galileo data (visible light data, in forward scattered light). Relative to the main ring, the halo is much less bright and more spatially confined at longer wavelengths and in backscattered light.

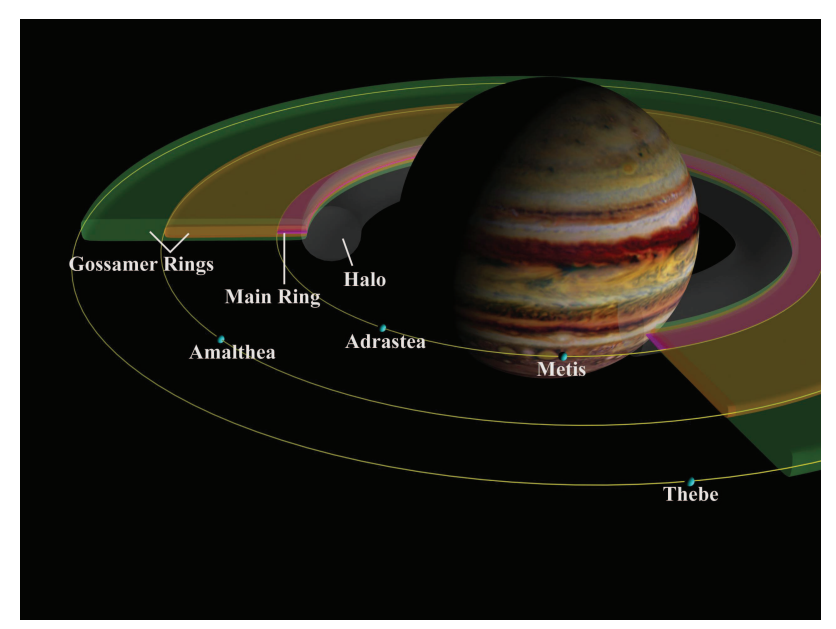

Figure 6.1 Schematic of Jupiters ring system, and its relation to the jovian ring-moons. (Ockert-Bell et al. 1999).

$\sim 179^{\circ}$ and $0.5 \mu \mathrm{m}-5 \mu \mathrm{m}$. In situ measurements of dust particles have further added to the characterization of ring material.

Since an excellent review of Jupiter's rings has been provided by Burns et al. (2004), in the present review we focus on observations published since that time, though we do provide some background to put the new material in context.

\subsection{The Main Ring and Halo}

\subsubsection{Main Ring Morphology}

The main ring is the most prominent component of the jovian ring system, especially when the rings are viewed in backscattered light, which is an indication that there is a relatively larger fraction of macroscopic material in this component of the rings compared to the fainter halo and gossamer rings. The total radial extent of the main ring is $\sim 7000 \mathrm{~km}$, with a $\sim 800$-km-wide bright annulus at its outer edge that has a sharp outer boundary near $129,100 \mathrm{~km}\left(1.81 R_{\mathrm{J}}\right)$. The ring's normal optical depth $\tau \approx$ a few $\times 10^{-6}$. Spacecraft and ground-based images have revealed detailed variations in the main ring's radial structure, such as shown in the visible-light image, Fig. 6.2 taken with the Galileo spacecraft in forward-scattered light. Radial scans through the

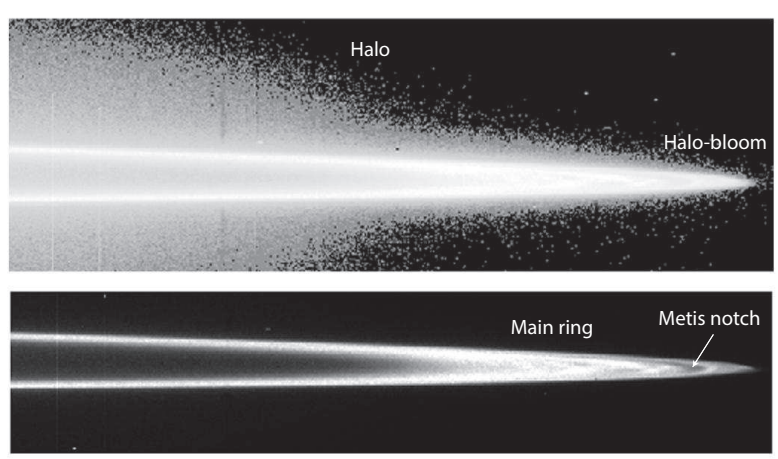

Figure 6.2 Galileo view of Jupiter's main ring at a phase angle of $176^{\circ}$. The top image has been stretched to show both the main ring and the halo, while the bottom image shows detailed patterns, such as the Metis gap, in the main ring. (Adapted from Ockert-Bell et al. (1999).

main ring annulus in both forward and backscattered light are shown in Fig. 6.3. Three bright radial bands are visible between the orbits of Metis and Adrastea and just outside Adreasta in the low-phase profile, but vanish in the corresponding high-phase images. These bands are thought to be the parent-body population.

Adrastea most likely clears a gap in the rings, while Metis controls the ring annulus inner edge. No structure is visible interior to the orbit of Metis, suggesting that there are no source bodies in this region; the region is composed entirely of dust.

Figure 6.4 shows several views of the jovian ring as observed with the Keck telescope at a wavelength of $2.2 \mu \mathrm{m}$ in backscattered light, when the rings were essentially edgeon. Because Jupiter's rings are optically thin, and assuming them to be cylindrically symmetric, one can invert the edgeon images by using an "onion-peel" deconvolution method to convert from integrated line-of-sight images, to radial profiles (e.g., Showalter et al. (1987); de Pater et al. (2008)). Starting from the outermost pixel in each row, the normal intensity of a narrow annulus that would produce the observed edge-on intensity is calculated, and then subtracted from each interior pixel in that row. This process is repeated until the entire image is inverted. Figure $6.4 \mathrm{c}-\mathrm{d}$ show images of the radial structure of the rings as obtained via this process, i.e., each row in this image represents a radial profile of the rings. To maximize the signal-to-noise in radial 


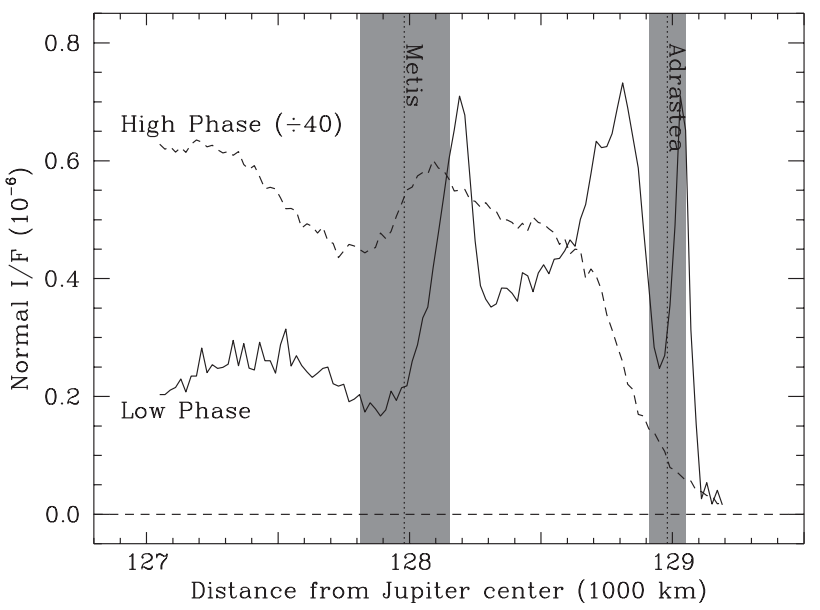

Figure 6.3 Comparison of radial profiles of the annulus of the main ring in both forward (high phase) and backscattered (low phase) light. In contrast to the forward scattered light profile, the backscattered profile shows much structure between the orbits of Metis and Adrastea, indicative of larger, parent bodies within the system. Vertical lines locate the orbital semimajor axes of Adrastea and Metis; the shaded vertical bands surrounding these orbital positions show the sweeping zones of the satellites. (From Burns et al. (2004), which was adapted from Showalter (2001)).

profiles, several rows in the edge-on images were integrated and onion-peeling was performed on these integrated edgeon profiles. Figure 6.5 shows the resulting radial profiles. The scans in panel a were obtained from a conventional "low-resolution" image (Fig. 6.4 ), and panels b-c from images taken with adaptive optics techniques (panel b from Fig. 6.4 , and panel c from an image with a higher spatial resolution).

Superposed on Fig. 6.5 is the Galileo visible-light lowphase radial profile. The Keck $2.2 \mu \mathrm{m}$ and Galileo profiles are in excellent agreement. The detailed structure in between and around the two moons remains unexplained. A small satellite was proposed to cause the low brightness between the satellites (Showalter et al. 1987), but the Keck data would have revealed moons $3-4 \mathrm{~km}$ in radius. New Horizons performed an exhaustive search for smaller embedded moons, with negative results down to a detection threshold of $\sim 0.5 \mathrm{~km}$ (Showalter et al. 2007). This implies a sharp break in the size distribution of small jovian moons, because the mass of the next-largest ring-moon, which remains unseen, has less than $\sim 10^{-4}$ of Adrastea's mass.

The New Horizons images did, however, reveal several clumps embedded within the rings. Because these clumps are spread out in longitude over $0.1^{\circ}-0.3^{\circ}$, the bright specks in Fig. 6.6 are not small moons, but rather distinct azimuthal clouds of material. These concentrations were not seen in forward-scattered light, so the clumps must be composed of larger-sized material. If this material is similar to that of the moon Adrastea, with a geometric albedo of 0.05, then the brightest clumps have an integrated surface area equivalent to a moon $0.5 \mathrm{~km}$ in radius. Previously, Throop et al. (2004) had found evidence for a single, longer $(\sim 1000 \mathrm{~km})$ clump

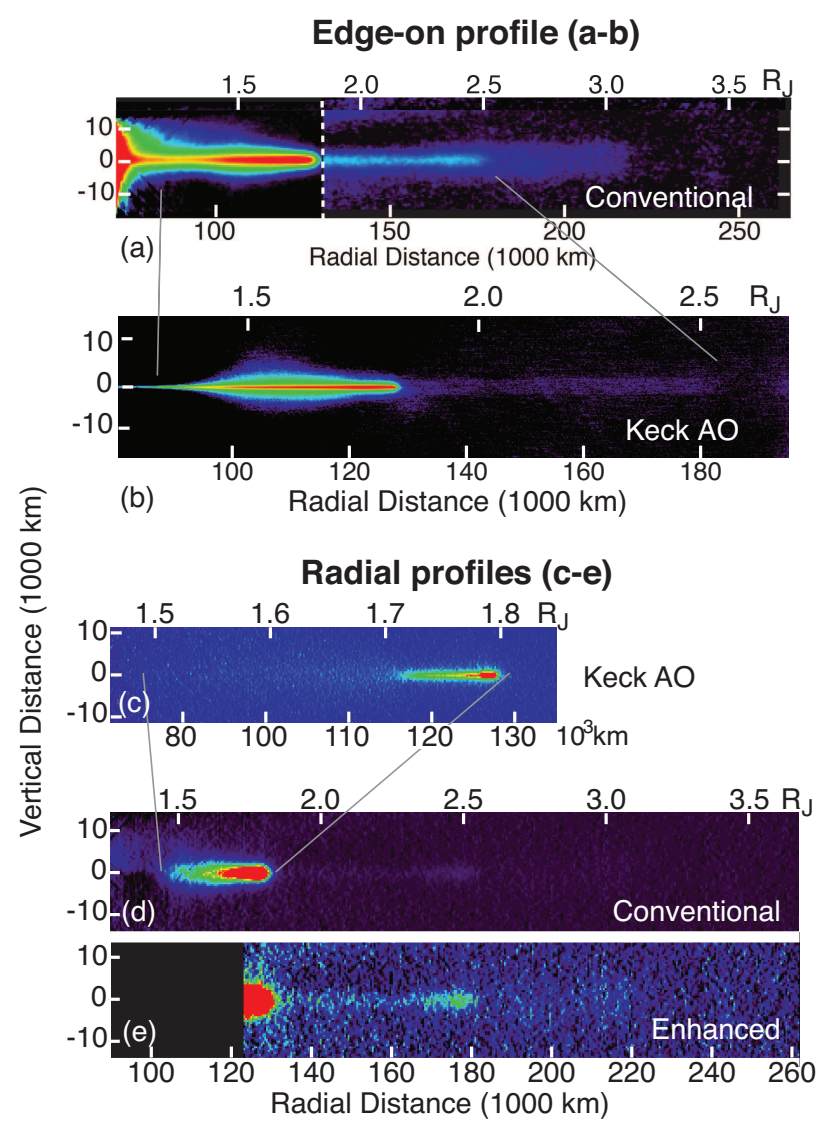

Figure 6.4 Images of Jupiter's ring system taken with the Keck telescope. a) Edge-on image of the rings at a wavelength of $2.2 \mu \mathrm{m}$; individual exposures were taken on 19 Dec. 2002 and 22 Jan. 2003. The main ring is visible in red, the halo in green, the inner gossamer ('Amalthea ring') in light-blue, and the outer gossamer ('Thebe ring') in dark blue. Note that the vertical thickness of the Thebe ring is much larger than that of the Amalthea ring. b) Edge-on image of the rings taken with the adaptive optics (AO) system at a wavelength of $2.2 \mu \mathrm{m}$ on 26 Jan. 2003. The main ring is visible in red, the halo in green, and the Amalthea ring in purple. The decreased intensity in the latter ring at the mid-plane is indicative of the same bright edges as seen in Fig. 6.10. c) Radial structure of the image in panel b, after applying an "onion"-peel technique, as described in the text. d) Radial structure obtained from the edge-on image in panel a. e) Same image as in panel d, but enhanced to show the Amalthea ring. The AO images have too low a signal-to-noise to extract the radial profile of the Amalthea ring. (Adapted from de Pater et al. (2008)).

in the Cassini images, but it remains unclear if these are observations of the same phenomenon.

\subsubsection{Halo}

The halo ring is an optically thin torus inward of Metis. The diffuse ring is visible in many images of the ring system, but no distinct features have been found within the ring. Interior to Metis, the ring's optical depth $\tau$ (Fig. 6.5 decreases steeply down to approximately the 3:2 Lorentz resonance at $122,400 \mathrm{~km}$. Inside of this resonance, the ring's brightness decreases more gradually, and seems to fade into the 

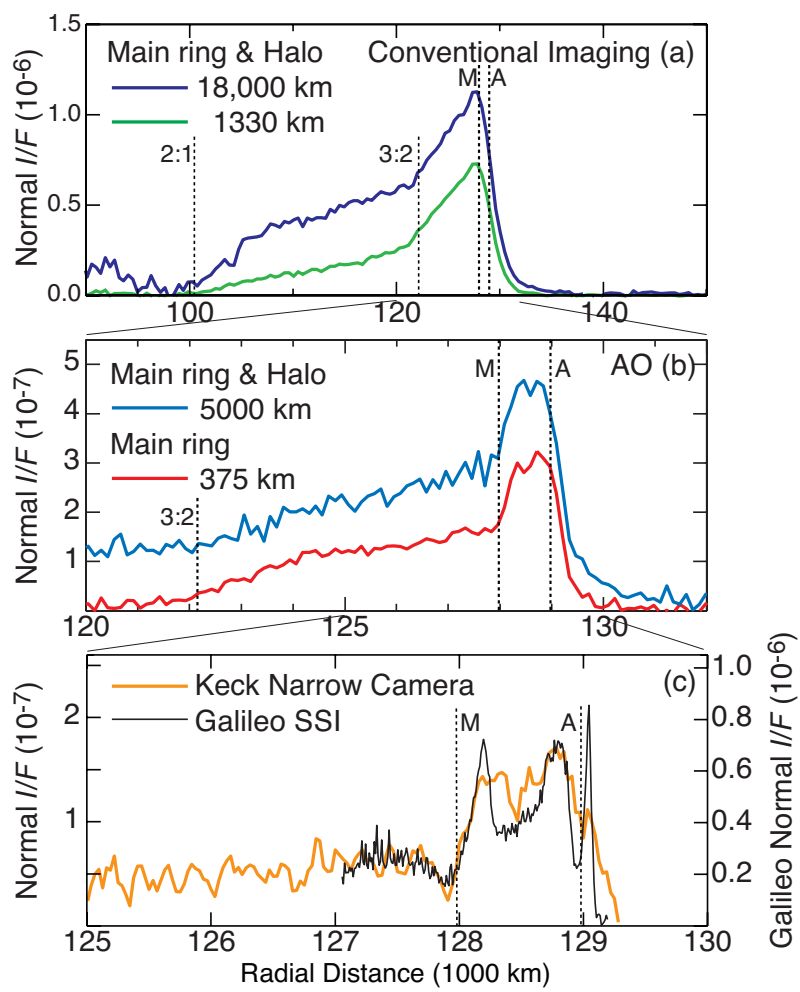

Figure 6.5 (a) Radial profiles through the main ring and halo, obtained by onion-peeling edge-on profiles obtained by integrating several rows in the image shown in Fig. 6.4 a, (amounting to vertical widths of $18,000 \mathrm{~km}$ and $1330 \mathrm{~km}$. The orbits of Metis (M) and Adrastea (A), as well as the 3:2 and 2:1 Lorentz resonances are indicated by dotted vertical lines. (b) Onion-peeled results from edge-on scans through the AO images in Fig. 6.4 b. The upper profile in panel b shows the resulting radial profile for an edge-on scan that was vertically integrated over both the main ring and halo (vertical width of $5000 \mathrm{~km}$ ).

The lower profile shows the result for just the main ring (vertical width of $375 \mathrm{~km}$ ). The orbits of Metis (M) and Adrastea (A), as well as the Lorentz 3:2 resonance, are indicated. (c) A high resolution radial profile through Jupiter's main ring, obtained by onion-peeling the edge-on profile from an image obtained with the highest resolution AO camera. The profile was smoothed radially over $0.03^{\prime \prime}$. Superposed is the visible light Galileo profile at low phase angles from Fig. 6.3 its intensity scale is shown on the y-axis on the right. (From de Pater et al. (2008)).

background inside the 2:1 Lorentz resonance at 101,200 km. Showalter (2001) showed that the halo's brightness varies as a power-law with height, $z$, above the ring plane. Closest to the ring plane, the brightness is proportional to $z^{-0.6}$, and several $1000 \mathrm{~km}$ up it follows a power law $z^{-1.5}$. Most of the halo's material is thus concentrated within just a few hundred $\mathrm{km}$ of the ring plane.

The Voyager and Galileo forward-scattered profiles suggest that the material interior to the main ring annulus is composed of micron-sized dust. This dust is produced from the parent bodies, and transported inwards by PoyntingRobertson drag. The particles within the halo probably had their inclinations increased by interactions with the planet's magnetic field at Jupiter's 3:2 Lorentz resonance. A second interaction with the 2:1 Lorentz resonance can perturb par- ticle orbits into Jupiter's atmosphere. Inside of the 2:1 resonance, the particle density is too low to be detectable from the ground. Galileo images have revealed a faint extended halo closer to the planet, but imaging becomes difficult because of the increase in telescopic stray light moving closer to Jupiter's limb (Throop et al., 2016).

Finally, exterior to the halo is a faint glow referred to as "halo bloom," which was only noted in Galileo images (Fig. 6.2 (Ockert-Bell et al., 1999). This material has not been studied further, but it may simply represent an outer, low-altitude component of the halo.

\subsubsection{Particle Size Distribution}

Spectra of the rings and variations in intensity with phase angle provide information on the composition and size distribution of the ring particles, both of which are tied to the source of the rings. Observations by a variety of spacecraft and Earth-based telescopes at backscatter all show the ring to be very red from $0.4-4 \mu \mathrm{m}$ (Fig. 6.7).

A variety of independent studies concluded that the color is indicative of the parent bodies' intrinsic color, and not an effect of scattering by small dust grains (de Pater et al., 1999. Throop et al. 2004, Wong et al., 2006). The parent bodies may be as red as Metis, a satellite that is $\sim 3$ times brighter at $2.1 \mu \mathrm{m}$ than at visual wavelengths, (de Pater et al. 1999). The $3.8 \mu \mathrm{m} \mathrm{1- \sigma}$ upper limit in Fig. 6.7 is consistent with a deep absorption feature that is also seen in a spectrum of Amalthea (Wong et al. 2006).

Images at 1.64 and $2.27 \mu \mathrm{m}$ further reveal that the halo contribution is stronger at the shorter wavelengths, possibly due to an intrinsic red color of the ring's parent bodies compared with the dusty halo component.

At high phase angles $\left(\alpha>120^{\circ}\right)$, the observed spectrum is dominated not by the intrinsic color of a body, but by the scattering properties of small dust grains. Throop et al. (2004) modeled the optical properties of dust to fit a merged dataset including observations from Galileo, Cassini, HST, and ground-based telescopes, over phase angles $\alpha=1^{\circ}-$ $179^{\circ}$ (Fig. 6.8). They modeled both the spectra and phase angle dependence with a power law differential particle size distribution:

$$
N(r) d r=N_{0} r^{-q} d r
$$

with $q \approx 2$ for particles with radii $r<15 \mu \mathrm{m}$, steepening to $q \approx 5$ for larger particles, with a maximum number density at $r \approx 15 \mu \mathrm{m}$. The red color of the rings, however, could not be explained by the ring's dust population alone, but required the distinctly red color of parent-sized material alluded to above. Throop et al. (2004) concluded that the main ring is composed of a combination of small grains with a normal optical depth $\tau \approx 4.7 \times 10^{-6}$, and larger bodies at $\tau \approx 1.3 \times 10^{-6}$. Since the phase curve is rather flat between $1^{\circ}$ and $\sim 130^{\circ}$, the authors concluded that the dust grains must be irregular-sized rather than spherical.

Most of the opacity due to large-sized material $(\tau \approx 1.1 \times$ $\left.10^{-6}\right)$ is contributed by a collection of parent bodies with radii over $\sim 5 \mathrm{~cm}$, according to a study by de Pater et al. (2008), who in addition to Keck and Galileo data also used 


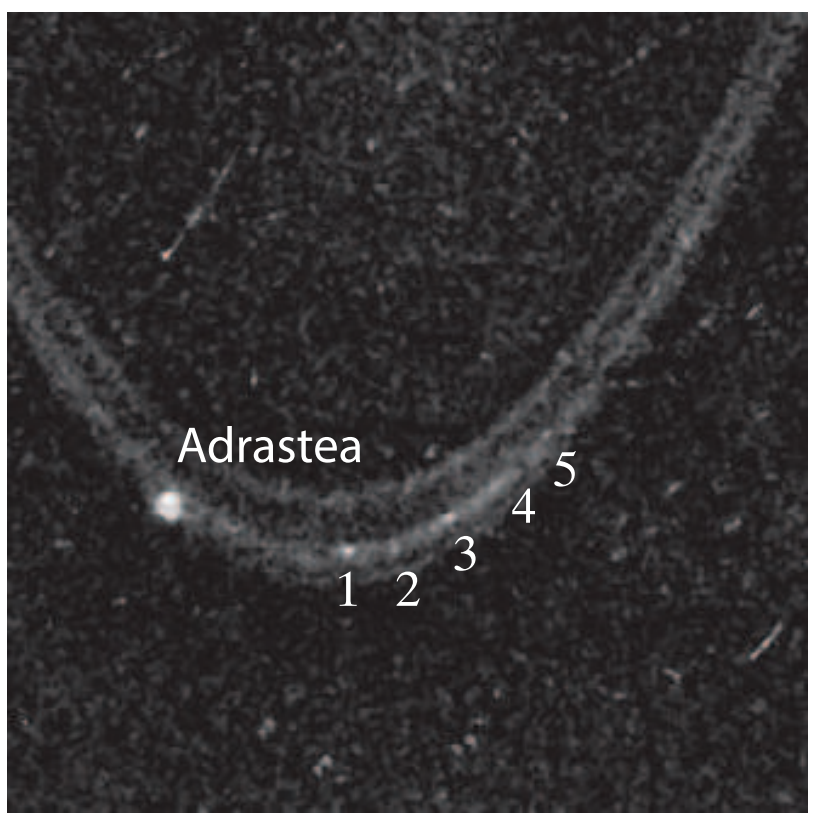

Figure 6.6 Image obtained with the New Horizons spacecraft en route to Pluto. The main ring annulus, including the central dip and the Adrastea gap, is clearly visible, as is Adrastea and the presence of 5 small clumps (1-5). (From (Showalter et al. 2007)).

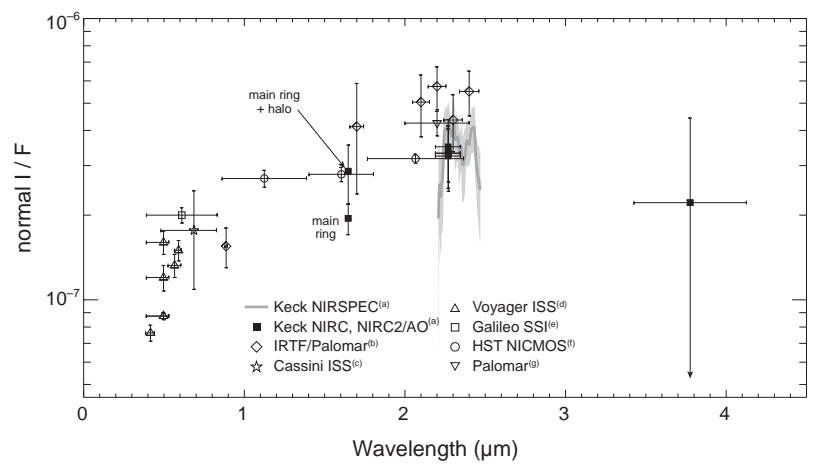

Figure 6.7 Spectral measurements of the back-scattered main ring reflectivity, after corrections for variations in the viewing geometry (by Throop et al. 2004). Horizontal bars indicate the wavelength range of each measurement. A Keck NIRSPEC spectrum is shown as a dark grey curve, with a light grey error envelope. The Keck data points at $1.64 \mu \mathrm{m}$ show the difference in $\mathrm{I} / \mathrm{F}$ when the halo is included or not; the $\mathrm{I} / \mathrm{F}$ for all other data points includes both contributions. The Keck data point near $3.8 \mu \mathrm{m}$ is a $1-\sigma$ upper limit. (Adapted from Wong et al. (2006)).

model fits of Jupiter's synchrotron radiation to microwave observations. The latter authors further suggested that this constitutes a fraction of $\sim 15 \%$ of the total optical depth in the main ring annulus. The additional optical depth is provided by dust grains tens-hundreds of $\mu \mathrm{m}$ across $(\tau \sim$ $3 \times 10^{-6}$ ), and another $\tau \sim 4 \times 10^{-6}$ is attributed to the small (sub)micron-sized grains that migrate radially inwards due to Poynting-Robertson drag. These small dust grains form the inward extension of the ring and the halo.

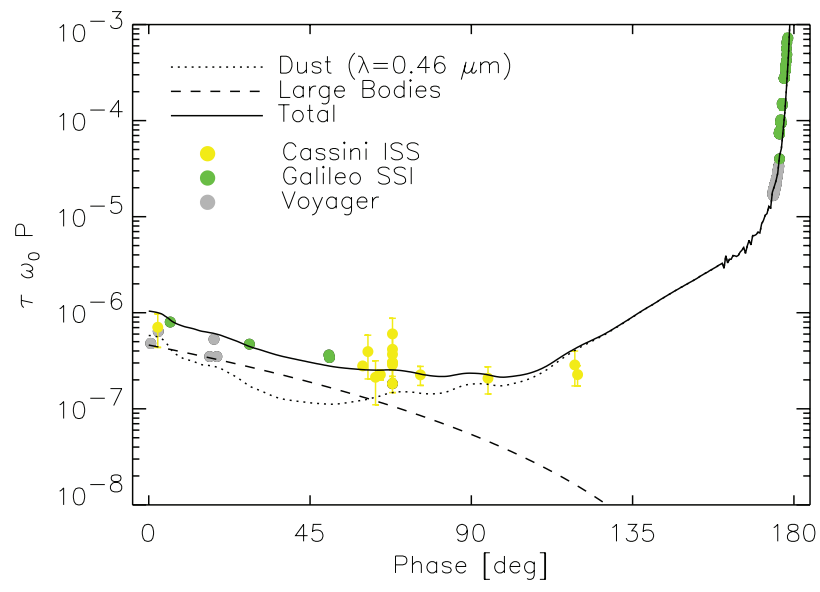

Figure 6.8 Cassini, Galileo and Voyager data as a function of phase angle, with superposed curves from Throop et al.'s (2004) best-fit non-spherical particle model at a wavelength of $0.46 \mu \mathrm{m}$ : the dotted line represents the dust component, the dashed line the large-body component, and the solid line the sum of the two. The small ripples in the dust phase curve are due to the finite number of oblateness values used in the model. (Throop et al. 2004)

\subsubsection{Models of the Jovian Ring System}

Two different models have been proposed for the formation of the jovian ring system. The first is the model proposed by Burns et al. (1999), which was briefly discussed in the introduction. In this model, dust is knocked off the various moons in the system, and subsequently evolves inwards under Poynting-Robertson drag. The orbit inclination and eccentricity are conserved in this process, and the particles essentially stay on their near-circular orbits. In the jovian plasma environment, small grains get charged and their orbital inclinations increase when they cross the $3: 2$ Lorentz resonance, producing the halo. In an alternative model, Horányi and Cravens (1996) proposed that the dust's inward migration is caused by plasma drag, which should lead to substantial changes in the eccentricity of a particle's orbit. The large eccentricities would result in a concentration of particles in a thin disk that extends radially from $\sim 80,000 \mathrm{~km}$ out to $\sim 140,000 \mathrm{~km}$. However, although observations do suggest the existence of a thin disk interior to the main ring, none of the observations show evidence for a thin disk that extends beyond the orbit of Adrastea.

\subsubsection{Impacts and Time Variability}

Recent studies have shown that the rings of Jupiter and Saturn can preserve a record of recent impacts (Hedman et al. 2011; Showalter et al. 2011). Subtle but distinctive spiral patterns can be traced back to the moment when the cloud of dust associated with a passing comet collided with the ring particles. Hedman et al. (2007) were the first to identify a pattern produced by a ring impact event. Cassini images of Saturn's innermost D ring revealed a clear pattern of periodic, alternating bright and dark bands determined to be vertical corrugations or "ripples". The wavelength of this pattern decreased over a timeframe of years, due to the 


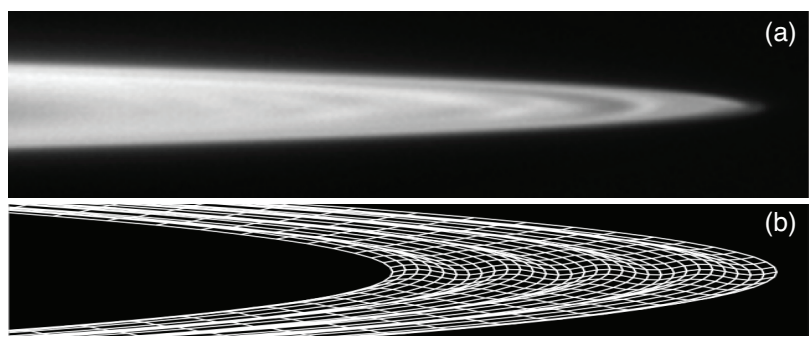

Figure 6.9 (a) After substantial contrast enhancement, Galileo image C0368974139 from November 1996 reveals subtle reversals of contrast across the ring ansa. (b) A cartoon illustration of how these reversals arise in nearly edge-on views of a corrugated ring. (see also Fig. 1 of Showalter et al. (2011))

differential regression rate of particles on orbits that initially shared a common node. Because the nodal rate is a known function of Saturn's gravity moments, the impact date could be pinpointed to late 1983 .

Showalter et al. (2011) showed that the Galileo spacecraft imaged a similar pattern of ripples in the jovian ring (Fig. 6.9). In this case they found a superposition of two patterns with different wavelengths, $1900 \mathrm{~km}$ and $600 \mathrm{~km}$. The stronger of these patterns had a vertical amplitude of $\sim 2.4 \mathrm{~km}$ and originated in mid-1994. Showalter et al. associated this "ring-tilting" event with the Shoemaker-Levy 9 (SL9) impacts in late July of that year. The secondary pattern originated four years earlier, and might have been caused by an earlier passage of SL9 through the jovian system. Furthermore, analysis of New Horizons images from 2007 revealed a pair of similar, but newer, ripple patterns in the ring, probably caused by smaller and previously unknown ring impacts during 2001 and 2003.

As the Solar System's largest planet, Jupiter receives sizable cometary impacts that are now being observed on a regular basis (Hueso et al., 2013). History also records four comets other than SL9 that have had very close brushes with Jupiter; in 1886, comet $\mathrm{P} /$ Brooks 2 may well have scored a direct hit on Jupiter's main ring. Kary and Dones (1996) showed that many comets are temporarily captured into a Jupiter orbit but never strike the planet. Any of these impacts and near-misses could potentially tilt the rings. Based on the four ripple patterns now observed, the time scale of cometary impacts producing observable ring displacements can be estimated at 1-2 per decade. Although the scars from planetary impacts dissipate on a time scale of weeks, each impact into the ring can produce a spiral pattern potentially observable for 5-10 years. As a result, the jovian ring may be the most effective "comet detector" in the Solar System.

These events may shed light on a long history of suggested, but often unconfirmed, variabilities in the jovian ring. For example, Voyager and Galileo clearly imaged "quadrant asymmetries" in the main ring (Showalter et al., 1987. Ockert-Bell et al., 1999), but Cassini and New Horizons did not (Throop et al. 2004). Cassini images showed a 1000-km clump orbiting within the ring (Throop et al., 2004), unlike anything seen before or since. During the New Horizons flyby, two clusters of very tiny clumps of unknown origin were seen within the main ring (Showalter et al. 2007), but

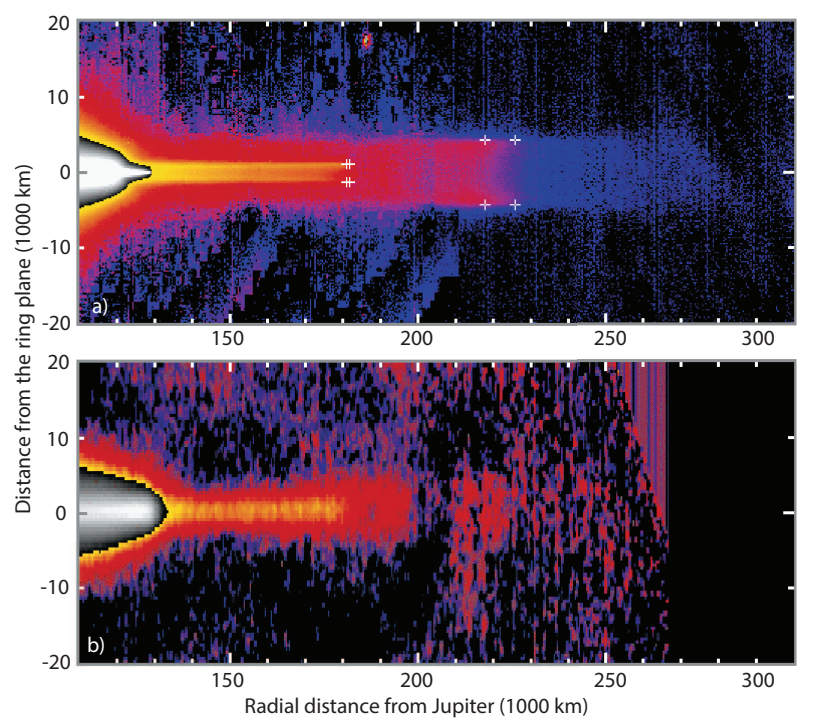

Figure 6.10 a) Mosaic of Jupiter's gossamer ring system obtained with the Galileo spacecraft at high phase angles, 177-179 (mosaic C10). Jupiter lies to the left with the main ring and halo in white, the Amalthea ring in yellow, the Thebe ring in red, and the Thebe extension in blue. The logarithm of the brightness is shown to reduce the dynamic range. The top and bottom edges of the gossamer rings are twice as bright as their central cores. White crosses mark the limits of Amalthea and Thebe's radial and vertical motions. b) Jupiter's gossamer rings at a back-scattering phase angle of $1.1^{\circ}$ taken at $2.27 \mu \mathrm{m}$ with Keck in August 1997, close to the time that the Earth crossed Jupiter's ring plane (the ring opening angle $B=0.17^{\circ}$ ). The geometric and color scale was chosen such as to match the Galileo image in panel a. The $0.60^{\prime \prime}$ seeing corresponds to a resolution of $1800 \mathrm{~km}$. The main ring, halo, and both gossamer components are apparent; hints of gossamer material are also visible further outward in the Keck data, albeit barely above the noise level, to the frame's edge at $\sim 257,000 \mathrm{~km}$. (Burns et al. 1999

these have not been seen in other data sets. Jupiter's high cometary influx may provide part of the explanation for why the jovian ring shows such variability.

\subsection{The Gossamer Rings}

The faint gossamer rings $\left(\tau \sim 10^{-7}\right)$ consist of two parts (Fig. 6.10): the 'Amalthea ring' lies immediately interior to the orbit of Amalthea (at $2.54 R_{\mathrm{J}}$ ); seen edge-on, this ring is almost uniform in brightness in both forward (panel a) and backscattered (panel b) light. Interior to Thebe (at 3.11 $R_{\mathrm{J}}$ ) is the 'Thebe ring', even fainter than the Amalthea ring. This ring extends inwards and overlaps with the Amalthea ring, and outwards to well beyond Thebe, out to $3.8 R_{\mathrm{J}}$, albeit at a brightness $\sim 10 \%$ of that of the main Thebe ring. As shown in Fig. 6.10a, the upper and lower edges of the gossamer rings are much brighter than their central cores. The vertical location of the peak brightness of each of the gossamer rings, as well as their vertical extent, corresponds to the maxima in the vertically projected distance of the 


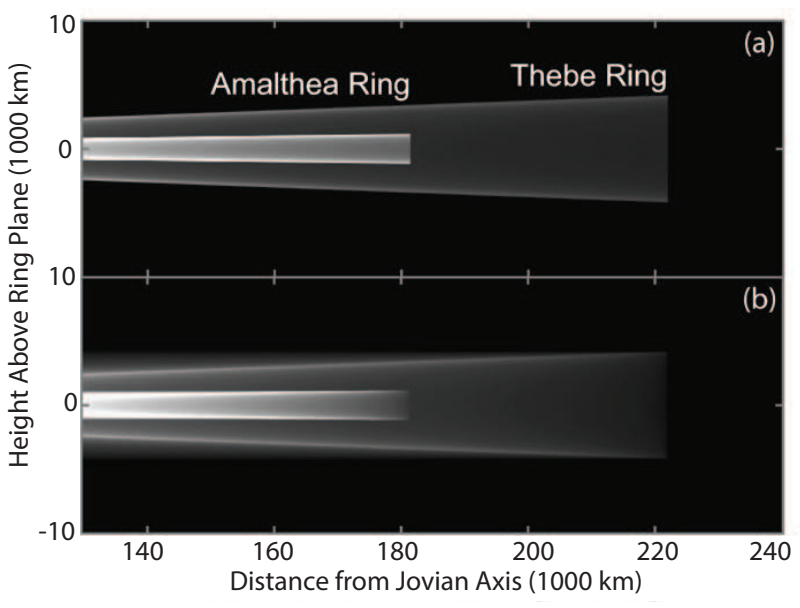

Figure 6.11 Model images of the gossamer rings. The overlapping Amalthea and Thebe rings are shown in (a) cross-sectional and (b) edge-on views. The density functions $h(r, z)$ have been scaled so that both rings have the same normal optical depths; the Thebe ring is fainter overall here only due to its greater vertical thickness. The images have been expanded vertically by a factor of two to better show the rings predicted vertical structure. (Showalter et al. 2008)

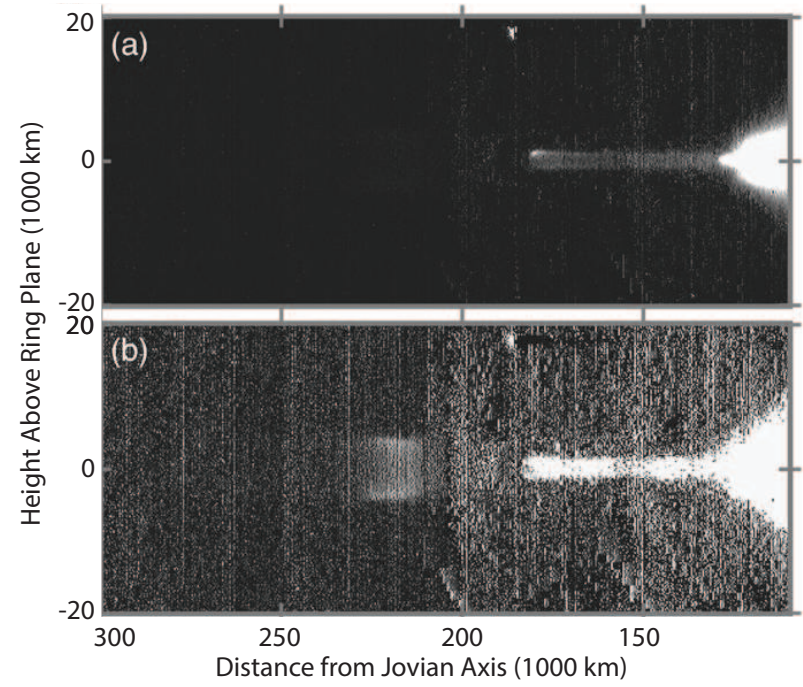

Figure 6.12 An onion-peeled image from Fig. 6.10 3 . The same image is shown with two enhancements, one emphasizing the Amalthea ring (a) and the other emphasizing the Thebe ring (b). The most surprising feature of Amalthea's ring is a bright peak in intensity near the northern tip. The Thebe ring may show an inner edge, but this is uncertain because it falls too close to the border between two images of the mosaic. (Showalter et al. 2008)

inclined orbits of Amalthea and Thebe, as indicated by the white crossess on the image. These characteristics imply that the particles originate from the bounding satellites, as postulated by the Burns et al. (1999) model.

Showalter et al. (2008) re-analyzed the Voyager and Galileo data, together with HST and Keck (Fig. 6.4 images. The Galileo data were taken in forward-scattered $\left(\alpha=170-178^{\circ}\right)$ and back-scattered $\left(\alpha \sim 0-11^{\circ}\right)$ light at vis-
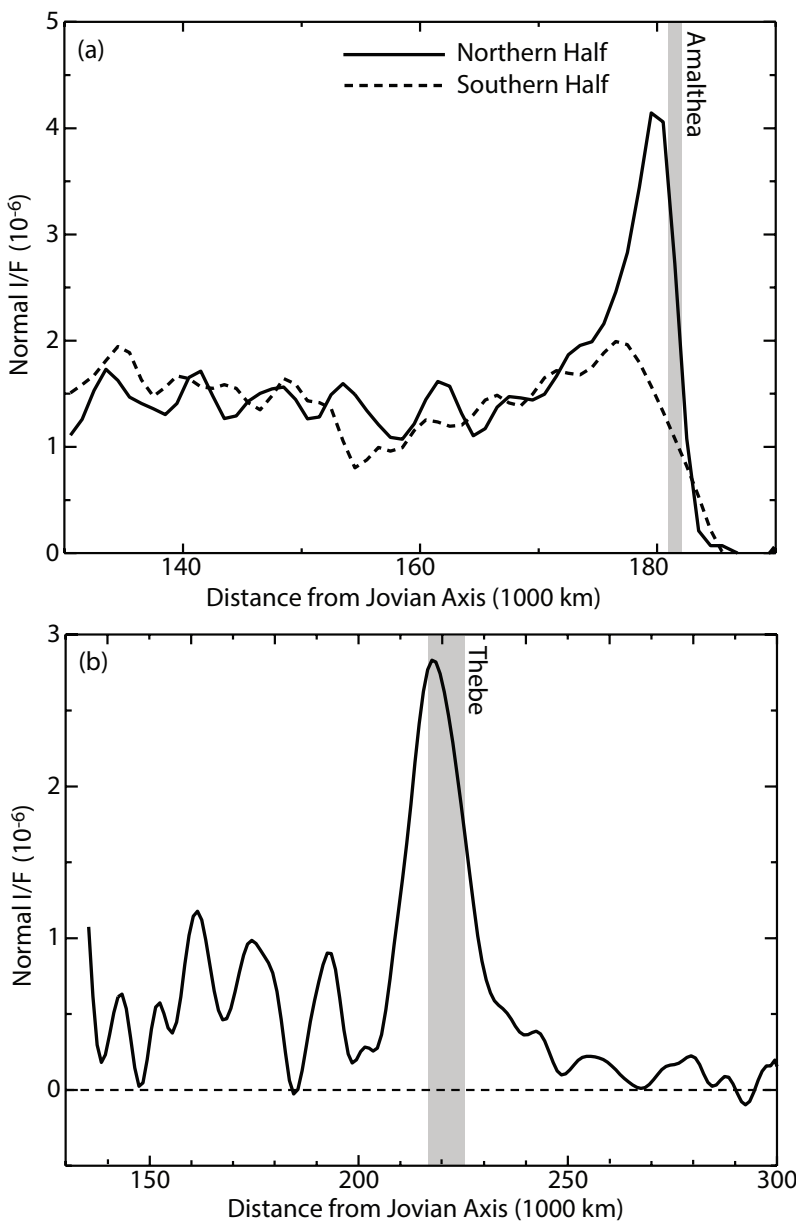

Figure 6.13 Onion-peeled radial profiles from the Galileo data. Before onion-peeling, to increase the signal-to-noise, an edge-on profile was created by vertically integrating rows on the image in Fig. 6.10 . a) Profiles through the northern and southern halves of the Amalthea ring: as in Fig. 6.12 the bright peak is only shown in the northern half. It is located close but interior to the orbit of Amalthea. b) The Thebe ring: a clear peak is visible just interior to Thebe's orbit. (Showalter et al. 2008)

ible and near-infrared wavelengths. The Keck and HST observations were taken in back-scattered light at near-infrared $(2.2 \mu \mathrm{m})$ and visible wavelengths, respectively. Just like the main ring and halo (Fig. 6.7), the gossamer rings are $\sim 3$ times brighter in the infrared than at visible wavelengths, indicative of a power law $q \approx 2$ in eq. 1 . An evaluation of Galileo and Voyager phase curves at high phase angles also indicates $q \sim 2-2.5$ (Showalter et al., 2008). The very low backscatter reflectivity of the ring, and a flat phase curve of the ring at low phase angles (as for the main rings, Fig. 6.8, suggests that the ring be composed of distinctly non-spherical particles (Showalter et al., 2008).

An onion-peeled image of the Keck data was shown in Fig. 6.4 panels $d$ and e, and an onion-peeled image of the Galileo mosaic (Fig. 6.10a) is shown in Fig. 6.12 These images look very different: in the Keck image (backscattered light at $2.3 \mu \mathrm{m}$ ), the Amalthea ring appears to be visible only near the satellite orbit, while the Galileo image (forward scattered visible-wavelength light) shows a structure 


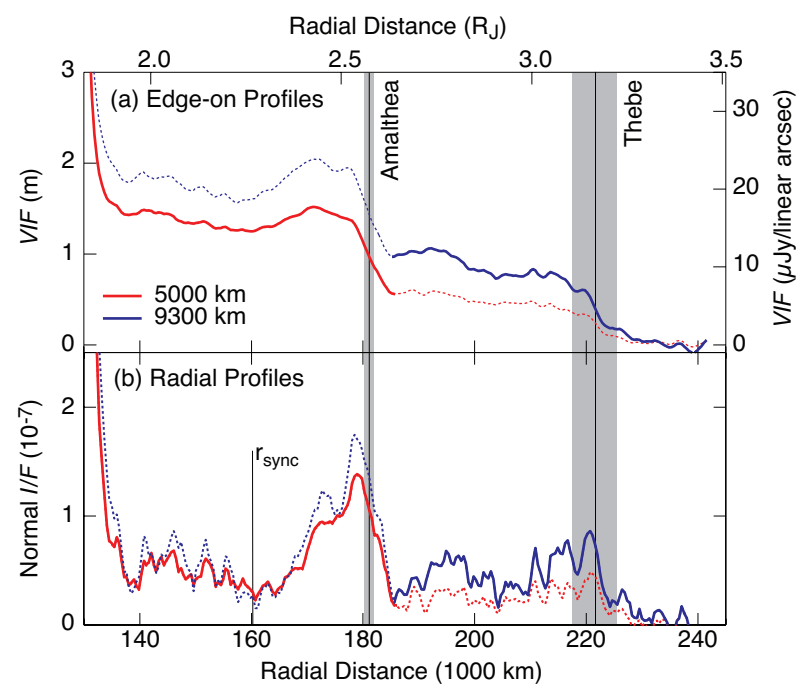

Figure 6.14 a) Edge-on profiles through the gossamer rings in Fig. 6.4 , integrated vertically over $\sim 1330 \mathrm{~km}, 5000 \mathrm{~km}$, and $9300 \mathrm{~km}$, as indicated. The orbits of Amalthea and Thebe are indicated by the vertical grey bars. b) Onion-peeled radial profiles from panel a. The orbits of Amalthea and Thebe, as well as the location of synchronous rotation $\left(r_{\text {sync }}\right)$ are indicated. The solid vs dashed lines indicate which profiles are most appropriate for which ring, as the vertical extent of the Thebe ring is about twice as large as for the Amalthea ring (de Pater et al. 2008).

very similar to that seen edge-on. The most striking feature in the latter image is the bright streak at the northern tip of Amalthea's ring. Onion-peeled radial profiles from the Galileo data are shown in Fig. 6.13, while Fig. 6.14 shows edge-on and onion-peeled radial profiles derived from the Keck edge-on image (Fig. 6.4 a) by integrating vertically over different heights. The Galileo-derived Amalthea ring profiles show that the bright feature is shifted inward from Amalthea's orbit by $\sim 1000 \mathrm{~km}$, and is roughly $\sim 10,000$ $\mathrm{km}$ wide. Considering the viewing geometry of the spacecraft and the actual position of Amalthea at the time of the observations, the detection of this material only along the northern edge was interpreted as being caused by a population of material trapped in Lagrangian points along Amalthea's orbit (Showalter et al. 2008). Figure 6.13p reveals a similar peak in Thebe's ring, also shifted inwards, but symmetric about the equator. Given Galileo's viewing geometry, Showalter et al. (2008) show that this peak can also be explained by material in a 1:1 resonance with Thebe.

The Keck-derived radial profiles are not that dissimilar from the Galileo profiles, in that a clear peak in brightness is seen just interior of Amalthea's orbit, although somewhat stronger (relative to the background profile) and broader than in the Galileo profiles. However, these data were integrated over 8-9 hours on different nights de Pater et al. 2008), which could possibly explain such differences. Moreover, as these data are taken in backscattered light, they are more sensitive to larger-sized material than the few up to $\sim 5 \mu \mathrm{m}$-sized dust grains sensed by the Galileo orbiter Krüger et al. 2009). Although the Thebe ring is clearly detected in the Keck profiles, the low signal-to-noise precludes an affirmative statement with regard to a potential concentration of particles near Thebe's orbit. If the minimum in brightness near the synchronous orbit location $(160,250 \mathrm{~km})$ is real, plasma drag may play a role in the physics of the particles' orbital migration.

Aside from these brightness enhancements, the overall profile of the gossamer rings in the Galileo data is quite uniform, supporting the model of dust evolving inward at a fixed rate under Poynting-Robertson drag. Figure 6.11 shows model images of this model, which indeed superficially matches the Galileo data quite well. Each ring is bounded at its outer edge by the orbit of its source moon, and has a vertical thickness that closely matches the thickness as expected for an inclined ring as projected on the sky. Also, each ring shows the expected concentrations of material near its upper and lower edges, and its vertical extent decreases with decreasing distance to the planet, in agreement with the data.

Although the Amalthea ring appears to be matched very well with the model, the Thebe ring's vertical profile is less compatible with the model, as the northern and southern edges are less sharp in the inner part of the Thebe ring than in the outer part, while both edges are relatively less bright than in the model. This suggests that the distribution of orbital inclinations of the particles appears to broaden while the grains move inward; they may receive kicks when they cross the strong 3:2 and 4:3 exterior Lorentz resonances (at 209,500 and $193,400 \mathrm{~km}$, resp.) on their journey inwards.

\subsection{In-situ Detection of Dust}

Near the end of its mission, the Galileo orbiter passed through the Thebe and Amalthea gossamer rings on November 5, 2002 and again on September 21, 2003. Impacts of numerous particles between 0.2 and $5 \mu \mathrm{m}$ in radius were detected, extending the size distribution downward to particles not apparent in visual imaging (Krüger et al., 2009). In addition to sensitivity to smaller particles, in-situ impact detections are also more sensitive to lower absolute number densities than direct imaging. Thus one of the main results of Galileo's passage through the ring region was the discovery that dust in the outer Thebe ring extends well beyond the limits of imaging to at least 5 Jupiter radii. The outer edge of the Thebe ring, therefore, is likely set by destabilizing gravitational perturbations from Io, located at $5.9 R_{\mathrm{J}}$.

Galileo also showed that the power-law size distribution measured optically in the main rings holds in the gossamer rings, at least for the micron-sized particles. Submicron particles follow this size distribution in the Thebe ring as well, but there is a notable excess of $0.2 \mu \mathrm{m}$ particles measured in the Amalthea ring (Krüger et al. 2009, Hamilton and Krüger, 2008). Finally, the incoming angles of impacting particles imply that orbital inclination angles for some grains extend up to at least $20^{\circ}$, far exceeding the $1^{\circ}$ tilt of large 
optically visible Thebe grains (Krüger et al. 2009 Hamilton and Krüger 2008).

These interesting observations can be explained as due to electromagnetic perturbations arising on orbiting dust grains (Hamilton and Krüger, 2008). Dust grains are electrically charged by interactions with sunlight and local plasma, so their motions are influenced by Jupiter's strong magnetic field (Burns et al., 2001, Burns et al., 2004). In particular, the change in a dust grain's electric charge during passage through Jupiter's shadow is especially important. As these changes occur once per orbit, the effect is resonant and is known as a shadow resonance. In its original formulation (Horanyi and Burns, 1991) the resonance excites orbital eccentricities, but there is also an analogous vertical shadow resonance that excites orbital inclinations (Hamilton and Krüger 2008). The eccentricity resonance can account for the outward extension of the Thebe ring seen both in imaging and in-situ while the narrower inclination resonance excites micron-sized grains to the high tilts implied by Galileo's in-situ detections (Hamilton and Krüger, 2008). The excess of submicron grains near Amalthea is likely due to its proximity to Jupiter's synchronous orbit where electromagnetic perturbations dramatically weaken. More details can be found in Hamilton and Krüger (2008) and in Chapter 12 of this book.

\subsection{Conclusions and Outlook}

The jovian ring has now been observed closely by seven spacecraft, starting with Pioneer 11's dust detector and including, very recently, Juno. With the inclusion of Earthbased data from HST and a number of large ground-based telescopes, the jovian ring system has been observed repeatedly and in some detail. From these disparate data sets a reasonably coherent picture of the rings has started to emerge. The rings are composed mostly of dust that is derived from meteorite impacts on the four ring-moons Thebe, Amalthea, Metis and Adrastea, as well as via impacts on and collisions between $\mathrm{cm}$ - and larger-sized material trapped in the main ring annulus and perhaps in 1:1 resonances with Amalthea and Thebe. The dust migrates inward due to PoyntingRobertson drag, as first suggested and modeled by Burns et al. (1999); their model seems to explain most of the ring's structure. Inward-migrating dust grains are "kicked" into a halo at the 2:3 Lorentz resonance; further inward, at the 1:2 Lorentz resonance, the inclinations of the particles are increased even more, and grains are ultimately lost into the atmosphere of Jupiter. Lorentz resonances in the Thebe ring are likely responsible for discrepancies between the Burns et al. (1999) model and observations of the Thebe ring. For example, the outward extension to the Thebe ring may be caused by small grains in Thebe's ring that receive large eccentricities due to a shadow resonance.

Nevertheless, observations of the ring remain few in number and are quite sparse in time coverage. Each observing period involves different viewing geometry and different instruments, often sensitive to different wavelengths. Most im- portantly, it involves a different epoch. Meanwhile, numerous ring features, from ripple patterns to clumps and asymmetries of various sorts, are time-variable. These phenomena are not possible to understand fully within such an episodic data set.

When the rings turn edge-on again in March 2021, multiwavelength observations with the James Webb Space Telescope (JWST) may help solve several still outstanding questions about the jovian system. However, we will probably not obtain a comprehensive understanding of this fascinating system until it can be scrutinized regularly and in detail by a Jupiter-orbiting spacecraft.

\section{Acknowledgements}

I.d.P. acknowledges partial support from NASA Planetary Astronomy (PAST) award NNX14AJ43G. M.R.S. acknowledges support from NASA?s Outer Planets Research Program through grant NNX14AO40G, and from Space Telescope Science Institute through program HST-GO-13414. Support for Program number HST-GO-13414 was provided by NASA through a grant from the Space Telescope Science Institute, which is operated by the Association of Universities for Research in Astronomy, Incorporated, under NASA contract NAS5-26555. D.P.H. acknowledges support from the Cassini Data Analysis Program Grant NNX15AQ67G. 


\section{REFERENCES}

Burns, J. A., Showalter, M. R., and Morfill, G. E. 1984. The ethereal rings of Jupiter and Saturn. Pages 200-272 of: Greenberg, R., and Brahic, A. (eds), IAU Colloq. 75: Planetary Rings.

Burns, J. A., Showalter, M. R., Hamilton, D. P., Nicholson, P. D., de Pater, I., Ockert-Bell, M. E., and Thomas, P. C. 1999. The Formation of Jupiter's Faint Rings. Science, 284(May), 1146.

Burns, J. A., Hamilton, D. P., and Showalter, M. R. 2001. Interplanetary Dust. Springer. Chap. Planetary Rings, pages 387-438.

Burns, J. A., Simonelli, D. P., Showalter, M. R., Hamilton, D. P., Porco, C. D., Throop, H., and Esposito, L. W. 2004. Jupiter's ring-moon system. Cambridge, UK: Cambridge University Press. Pages 241-262.

de Pater, I., and Lissauer, J. J. 2015. Planetary Sciences. Cambridge, UK: Cambridge University Press.

de Pater, I., Showalter, M. R., Burns, J. A., Nicholson, P. D., Liu, M. C., Hamilton, D. P., and Graham, J. R. 1999. Keck Infrared Observations of Jupiter's Ring System near Earth's 1997 Ring Plane Crossing. Icarus, 138(Apr.), 214-223.

de Pater, I., Showalter, M. R., and Macintosh, B. 2008. Keck observations of the 20022003 jovian ring plane crossing. Icarus, 195(May), 348-360.

Fillius, R. W., McIlwain, C. E., and Mogro-Campero, A. 1975. Radiation belts of Jupiter - A second look. Science, 188(May), 465-467.

Hamilton, D. P., and Krüger, H. 2008. The sculpting of Jupiter's gossamer rings by its shadow. Nature, 453(May), 72-75.

Hedman, M. M., Burns, J. A., Showalter, M. R., Porco, C. C., Nicholson, P. D., Bosh, A. S., Tiscareno, M. S., Brown, R. H., Buratti, B. J., Baines, K. H., and Clark, R. 2007. Saturn's dynamic D ring. Icarus, 188(May), 89-107.

Hedman, M. M., Burns, J. A., Evans, M. W., Tiscareno, M. S., and Porco, C. C. 2011. Saturn's Curiously Corrugated C Ring. Science, 332(May), 708.

Horanyi, M., and Burns, J. A. 1991. Charged dust dynamics Orbital resonance due to planetary shadows. JGR, 96(Nov.), 19.

Horányi, M., and Cravens, T. E. 1996. The structure and dynamics of Jupiter's ring. Nature, 381(May), 293-295.

Hueso, R., Pérez-Hoyos, S., Sánchez-Lavega, A., Wesley, A., Hall, G., Go, C., Tachikawa, M., Aoki, K., Ichimaru, M., Pond, J. W. T., Korycansky, D. G., Palotai, C., Chappell, G., Rebeli, N., Harrington, J., Delcroix, M., Wong, M., de Pater, I., Fletcher, L. N., Hammel, H., Orton, G. S., Tabe, I., Watanabe, J., and Moreno, J. C. 2013. Impact flux on Jupiter: From superbolides to large-scale collisions. $A \& A, \mathbf{5 6 0}$ (Dec.), A55.
Kary, D. M., and Dones, L. 1996. Capture Statistics of ShortPeriod Comets: Implications for Comet D/Shoemaker-Levy 9. Icarus, 121(June), 207-224.

Krüger, H., Hamilton, D. P., Moissl, R., and Grün, E. 2009. Galileo in-situ dust measurements in Jupiter's gossamer rings. Icarus, 203(Sept.), 198-213.

Ockert-Bell, M. E., Burns, J. A., Daubar, I. J., Thomas, P. C., Veverka, J., Belton, M. J. S., and Klaasen, K. P. 1999. The Structure of Jupiter's Ring System as Revealed by the Galileo Imaging Experiment. Icarus, 138(Apr.), 188-213.

Showalter, M. R., Hamilton D. P. Burns J. A. de Pater I. Simonelli D. P. 2001. Structure of Jupiter's main ring and halo from Galileo SSI and Earth-based images. Pages 100101 of: Planet, Satellites and Magnetosphere.

Showalter, M. R., Burns, J. A., Cuzzi, J. N., and Pollack, J. B. 1985. Discovery of Jupiter's 'gossamer' ring. Nature, 316(Aug.), 526-528.

Showalter, M. R., Burns, J. A., Cuzzi, J. N., and Pollack, J. B. 1987. Jupiter's ring system - New results on structure and particle properties. Icarus, 69(Mar.), 458-498.

Showalter, M. R., Cheng, A. F., Weaver, H. A., Stern, S. A., Spencer, J. R., Throop, H. B., Birath, E. M., Rose, D., and Moore, J. M. 2007. Clump Detections and Limits on Moons in Jupiter's Ring System. Science, 318(Oct.), 232.

Showalter, M. R., de Pater, I., Verbanac, G., Hamilton, D. P., and Burns, J. A. 2008. Properties and dynamics of Jupiter's gossamer rings from Galileo, Voyager, Hubble and Keck images. Icarus, 195(May), 361-377.

Showalter, M. R., Hedman, M. M., and Burns, J. A. 2011. The Impact of Comet Shoemaker-Levy 9 Sends Ripples Through the Rings of Jupiter. Science, 332(May), 711.

Smith, B. A., Soderblom, L. A., Beebe, R., Boyce, J., Briggs, G., Carr, M., Collins, S. A., Johnson, T. V., Cook, II, A. F., Danielson, G. E., and Morrison, D. 1979a. The Galilean satellites and Jupiter - Voyager 2 imaging science results. Science, 206(Nov.), 927-950.

Smith, B. A., Soderblom, L. A., Johnson, T. V., Ingersoll, A. P., Collins, S. A., Shoemaker, E. M., Hunt, G. E., Masursky, H., Carr, M. H., Davies, M. E., Cook, A. F., Boyce, J. M., Owen, T., Danielson, G. E., Sagan, C., Beebe, R. F., Veverka, J., McCauley, J. F., Strom, R. G., Morrison, D., Briggs, G. A., and Suomi, V. E. 1979b. The Jupiter system through the eyes of Voyager 1. Science, 204(June), 951-957.

Throop, H. B., Porco, C. C., West, R. A., Burns, J. A., Showalter, M. R., and Nicholson, P. D. 2004. The jovian rings: new results derived from Cassini, Galileo, Voyager, and Earthbased observations. Icarus, 172(Nov.), 59-77.

Throop, H. B., Showalter, M. R., Dones, H. C., Hamilton, D. P., Weaver, H. A., Cheng, A. F., Stern, S. A., Young, L., Olkin, C. B, and New Horizons Science Team. 2016. New Horizons 
Imaging of Jupiter's Main Ring. AAS/Division for Planetary Sciences Meeting, 48(Oct.).

van Allen, J. A., Randall, B. A., Baker, D. N., Goertz, C. K., Sentman, D. D., Thomsen, M. F., and Flindt, H. R. 1975. Pioneer 11 observations of energetic particles in the Jovian magnetosphere. Science, 188(May), 459-462.

Wong, M. H., de Pater, I., Showalter, M. R., Roe, H. G., Macintosh, B., and Verbanac, G. 2006. Ground-based near infrared spectroscopy of Jupiter's ring and moons. Icarus, 185(Dec.), 403-415. 